\title{
La discapacidad visual y la ceguera en la pandemia del COVID-19.
}

\author{
Visual impairment and blindness in the COVID-19 pandemic
}

Jorge Luis Chin-Wong ${ }^{1, a}$

Se calculan alrededor de 70 millones de personas con discapacidad en América Latina y el Caribe y que experimentan discriminaciones múltiples y simultáneas que han empeorado a raíz de la crisis sanitaria por el COVID-19. La discapacidad es una causa y, a su vez, una consecuencia de la pobreza (1). Dentro de este grupo, las personas que sufren discapacidad visual reciben un mayor impacto en lo económico y social que se mide no sólo por el gasto en medicamentos o terapias visuales, si no también, en la limitación del paciente para participar de labores económicamente productivas teniendo en cuenta las circunstancias actuales de confinamiento total o parcial debido a la pandemia.

Según el informe mundial de la visión de la OMS, se estima que en el mundo hay alrededor de 2200 millones de personas con deterioro de la visión cercana o distante. Al menos en el $50 \%$ de ellos, el deterioro visual podría haberse evitado. Entre esos casos se encuentran aquellos debido a errores de refracción no corregidos (88,4 millones), cataratas (94 millones), glaucoma (7,7 millones), opacidades corneales (4,2 millones), retinopatía diabética (3,9 millones) y tracoma (2 millones), así como deterioro de la visión cercana causado por presbicia no corregida (826 millones) (2).

En el Perú, el estudio realizado por Campos et al (3) muestra que la prevalencia de ceguera en el país en mayores de 50 años es de $2 \%$,, en el cual la catarata fue la causa principal de ceguera $(58,0 \%)$, seguida por el glaucoma $(13,7 \%)$ y la degeneración macular relacionada con la edad $(11,5 \%)$. Además se encontró que los errores de refracción no corregidos fueron la principal causa de deficiencia visual moderada $(67,2 \%)$.

A un año del inicio del estado de emergencia, los profesionales de la salud nos enfrentamos no sólo al COVID-19, también a todas las otras enfermedades "no COVID-19" que tienen impacto en la morbilidad y mortalidad de la población y que causan la muerte de más de 40 millones de personas en el mundo. Muchas personas que necesitan tratamiento para enfermedades como cáncer, padecimientos cardiovasculares y diabetes no han recibido los cuidados y medicinas que precisan desde que empezó la pandemia. La situación es alarmante porque las personas que viven con estos padecimientos corren un mayor riesgo de muerte si adquieren el coronavirus. Según una encuesta realizada por la OMS el 53\% de los países han interrumpido parcial o totalmente los programas de tratamiento de la hipertensión, la diabetes y las complicaciones relacionadas con esta enfermedad. Del mismo modo, el tratamiento para el cáncer se ha visto afectado en $42 \%$ de los países y, en el caso de las emergencias cardiovasculares, la afectación abarca al $31 \%$ de los países ${ }^{(4)}$. Sin embargo, también se afectó el diagnóstico y tratamiento de enfermedades visuales, debido a que se restringieron las atenciones y las cirugías oftalmológicas a nivel público y privado. Además se debe considerar que los oftalmólogos son especialistas médicos que han demostrado ser susceptibles del contagio por contacto en consulta externa con pacientes COVID-19 ${ }^{(5,6)}$, por lo que varios suspendieron sus actividades por presentar comorbilidades.

Los errores refractivos adquieren importancia en este momento por el empleo de aulas y clases virtuales, así como por el trabajo remoto. Asociado a estas condiciones está el síndrome de ojo seco, el cual se ha convertido en una de las causas más frecuentes de consulta oftalmológica. La prescripción de anteojos son atenciones presenciales, y por ende, limitadas por la actual situación.

El tratamiento de las causas más frecuentes de ceguera: la catarata y el glaucoma han sido afectadas por la restricción de las consultas y cirugías en los hospitales públicos. Los pacientes con catarata presentan avance de su enfermedad, salvo que se operen en forma particular, y los pacientes de glaucoma requieren un adecuado control de la presión intraocular para evitar su progresión hacia la ceguera irreversible.

1. Servicio de oftalmología, Hospital Regional Lambayeque, Chiclayo, Perú

a. Especialista en Oftalmología, Microcirugía de Segmento Anterior 
Durante la pandemia, se han realizado teleconsultas a pacientes con este diagnóstico, con la finalidad de prescribir hipotensores oculares para su tratamiento, pero con la gran limitante de no poder monitorear en tiempo real la presión intraocular. Los pacientes que no tienen acceso a estos medicamentos, pueden desarrollar ceguera y discapacidad visual importante, si no son atendidos a tiempo. La retinopatía diabética, es otra causa importante de ceguera irreversible, que ocurre como consecuencia de un mal control metabólico de los pacientes con diabetes y que progresa a una fase proliferativa asociada a mala visión por complicaciones serias e irreversibles. En la actual coyuntura es clave el mantener a los pacientes con enfermedades crónico degenerativas bajo control medicamentoso adecuado.

Otro tema sensible es la discapacidad visual en la población infantil. La catarata congénita y la retinopatía del prematuro son causas importantes de ceguera infantil, y al igual que en la población adulta, los errores de refracción no corregidos siguen siendo la causa principal de deterioro de la visión entre los niños. Los infantes con deterioro de la visión grave de inicio temprano pueden sufrir retrasos en el desarrollo motor, lingüístico, emocional, social y cognitivo, con consecuencias para toda la vida. Los niños en edad escolar que presentan deterioro de la visión también pueden manifestar niveles más bajos de rendimiento académico ${ }^{(2,7)}$. En el Hospital Regional Lambayeque reanudamos el tamizaje de retinopatía del prematuro en niños nacidos de madres positivas a COVID-19 en nuestro hospital y su seguimiento por consultorio externo, por la importancia que tiene este diagnóstico para el desarrollo futuro de nuestra población pediátrica.

Finalmente, esta pandemia para las personas ciegas ha significado un desafío de dimensiones considerables en la medida en que se tiene que hacer frente a nuevas barreras para su movilidad, su vida independiente y su salud mental. La pandemia ha implicado un incremento de las barreras a la movilidad y transporte de estas personas, dificultando su acceso a artículos y servicios esenciales, aumentando su dependencia de otras personas. Los hábitos de higiene esenciales, como el uso de mascarillas, la distancia de seguridad o no tocar superficies son desafíos adicionales que dificultan su capacidad de orientación y sensación de seguridad al desplazarse. Asimismo, el acceso a otras actividades de tiempo libre y recreativas, debido al cierre de los espacios públicos o a las limitaciones de muchas plataformas online para su accesibilidad a personas con ceguera, ha supuesto una limitación adicional a sus posibilidades de entretenimiento. De esta manera, los sentimientos de frustración, ansiedad, ira, baja autoestima y desmotivación causados por la pérdida de autonomía e independencia han sido frecuentemente informados por estas personas durante este periodo, acentuando su sensación de aislamiento y desconexión social ${ }^{(8)}$.

En conclusión, la discapacidad visual y la ceguera en este contexto de pandemia, han aumentado la brecha existente para su inclusión social. Los tratamientos, procedimientos o cirugías que se realizaban antes de la pandemia, que de por sí ya eran insuficientes a pesar del esfuerzo del Gobierno con el Decreto Legislativo N¹468 (que establece disposiciones de prevención y protección para las personas con discapacidad ante la emergencia sanitaria ocasionada por el COVID-19) emitido en Abril del año 2020; y del Plan Nacional de Salud Ocular y Prevención de la Ceguera, han sido suspendidos con el consecuente perjuicio de la población usuaria, quienes se ven resignados a esperar la reactivación de los servicios de oftalmología, si es que no tienen recursos económicos que les permitan atenderse de forma particular. Es de esperar que el número de personas con discapacidad visual o con ceguera aumente una vez superada la emergencia sanitaria, junto con problemas en la esfera emocional que pueden causar sufrimiento psicológico, dificultando su inclusión en la sociedad.

\section{REFERENCIAS BIBLIOGRÁFICAS}

1. Meresman S, Ullmann H, "COVID-19 y las personas con discapacidad en América Latina: mitigar el impacto y proteger derechos para asegurar la inclusión hoy y mañana", serie Políticas Sociales, N²37 (LC/ TS.2020/122), Santiago, Comisión Económica para América Latina y el Caribe (CEPAL), 2020.

2. Vision Loss Expert Group of the Global Burden of Disease Study. Causes of blindness and vision impairment in 2020 and trends over 30 years: evaluating the prevalence of avoidable blindness in relation to "VISION 2020: the Right to Sight". Lancet Global Health 2020. doi.org/10.1016/ S2214-109X(20)30489-7

3. Campos B, Cerrate A, Montjoy E, Dulanto V, Gonzales C, Tecse A, Pariamachi A, Lansingh, Van C., Dulanto V, Minaya J, Silva J, Limburg H. (2014) Prevalencia y causas de ceguera en Perú: encuesta nacional. Rev Panam Salud Publica;36(5) 283-289,nov. 2014. acceso online http://www.scielosp.org/scielo.phpscript=sci_arttext\&pid=S1020-49892014001000001

4. World Health Organization. The impact of the COVID-19 pandemic on noncommunicable disease resources and services: results of a rapid assessment. Geneva: World Health Organization; 2020. https://www.who. int/teams/noncommunicable-diseases/covid-19\&publication=ncds-covid-rapid-assessment

5. Kuo IC, \& O'Brien TP (2020). COVID-19 and ophthalmology: an underappreciated occupational hazard. Infection control and hospital epidemiology, 41(10), 1207-1208. https://doi.org/10.1017/ice.2020.238

6. Bressler NM. Ophthalmology and COVID-19. JAMA. 2020;324(12):11431144. doi:10.1001/jama.2020.17595

7. Vision Loss Expert Group of the Global Burden of Disease Study. Trends in prevalence of blindness and distance and near vision impairment over 30 years: an analysis for the Global Burden of Disease Study. Lancet Global Health 2020. doi: 10.1016/S2214-109X(20)30425-3

8. World Blind Union. WBU Global Report - COVID-19, Amplifying Voices, Our Lives, Our Say 2020. Acceso online: http://www.infocop.es/pdf/amplifying_voices.pdf 